\title{
Participants in the Symposium
}

Richard H. Backus

Gregory Bateson

Keller Breland

René-Guy Busnel

David K. Caldwell

Melba C. Caldwell

William H. Dawbin

John J. Dreher

W. H. Dudok van Heel

Albin Dziedzic

William J. L. Felts

N. J. Flanigan

F. C. Fraser

Robert S. Gales

Charles O. Handley, Jr.

Carl L. Hubbs

Tadayoshi Ichihara
Woods Hole Oceanographic Institution, Woods Hole, Massachusetts

Communications Research Institute, St. Thomas, Virgin Islands

President, Animal Behavior Enterprises, Inc., Hot Springs, Arkansas

Directeur, Laboratoire d'Acoustique Animale, Jouyen-Josas, Seine et Oise, France

Curator of Marine Zoology, Los Angeles County Museum, Los Angeles, California

Los Angeles County Museum, Los Angeles, California

Senior Lecturer in Zoology, University of Sydney, Sydney, Australia

Lockheed-California Corporation, Burbank, California

Aboard MV Cachelot, Espelervaart, Emmeloord, Netherlands

International Group of the Laboratoire d'Acoustique Animale, Strib-Havn, Denmark

Department of Anatomy, Medical School, University of Minnesota, Minneapolis, Minnesota

Biology Department, St. Norbert College, West De Pere, Wisconsin

Keeper of Zoology, Department of Zoology, British Museum (Natural History), London, England

Head, Listening Division, U.S. Navy Electronics Laboratory, Point Loma, California

Associate Curator, Division of Mammals, Smithsonian Institution, Washington, D.C.

Professor, Scripps Institution of Oceanography, University of California, San Diego

Whales Research Institute, Tokyo, Japan 
Laurence Irving

Age Jonsgård

John Kanwisher

Winthrop N. Kellogg

Lawrence Kruger

Thomas G. Lang

John C. Lilly

R. Stuart Mackay

N. A. Mackintosh

\section{Harrison Matthews}

Joseph Curtis Moore

Masaharu Nishiwaki

Kenneth S. Norris

Hideo Omura

Peter E. Purves

Carleton Ray

Irving Rehman

F. W. Reysenbach de Haan

Charles E. Rice

Dale W. Rice

William E. Schevill

Per F. Scholander

David E. Sergeant
Professor, Laboratory of Zoophysiology, University of Alaska, College, Alaska

Associate Professor, Institute of Marine Biology, and Deputy Director, State Institute of Whale Research, Oslo, Norway

Woods Hole Oceanographic Institution, Woods Hole, Massachusetts

Stanford Research Institute, Menlo Park, California Associate Professor of Anatomy, Brain Research Institute, University of California, Los Angeles

U.S. Naval Ordnance Test Station, Pasadena, California

Director, Communication Research Institute, Miami, Florida

Professor, Medical Physics and Space Sciences Laboratory, University of California, Berkeley

Officer-in-Charge, Whale Research Unit, National Institute of Oceanography, British Museum (Natural History), London, England

Scientific Director, Zoological Society of London, England

Curator of Mammals, Chicago Natural History Museum, Chicago, Illinois

Whales Research Institute, Tokyo, Japan

Associate Professor of Zoology, University of California, Los Angeles

Director, Whales Research Institute, Tokyo, Japan Senior Experimental Officer, Department of Zoology, British Museum (Natural History), London, England

Associate Curator, New York Aquarium, New York Zoological Society, New York

School of Medicine, University of Southern California, Los Angeles

Lutherian Hospital, Eindhoven, Netherlands

Stanford Research Institute, Menlo Park, California

U.S. Department of Interior, Bureau of Commercial Fisheries, Seattle, Washington

Woods Hole Oceanographic Institution, Woods Hole, Massachusetts

Professor of Physiology, Scripps Institution of Oceanography, University of California, San Diego Fisheries Research Board of Canada, Arctic Unit. Montreal, Canada 
William C. Shaw

George G. Silberberg

E. J. Slijper

James M. Snodgrass

Winston H. Starks

John C. Steinberg

Gunnar Sundnes

Margaret C. Tavolga

William A. Watkins

F. G. Wood, Jr.
Professor of Physics, Science and Technology Division, Southern Illinois University, East St. Louis, Illinois

U.S. Naval Ordnance Test Station, China Lake, California

Professor of General Zoology, University of Amsterdam, Amsterdam, Holland

Division Head, Special Developments, Scripps Institution of Oceanography, University of California, San Diego

Advanced Research and Special Devices Laboratory, American Electronics Laboratories, Colmar, Pennsylvania

Professor, Institute of Marine Science, University of Miami, Miami, Florida

Fiskeridirektoratets Havforskningsinstitutt, Bergen, Norway

Department of Animal Behavior, American Museum of Natural History, New York, and Department of Biological Sciences, Fairleigh Dickinson University, Teaneck, New Jersey

Woods Hole Oceanographic Institution, Woods Hole, Massachusetts

U.S. Naval Missile Center, Point Mugu, California 
